\title{
Supplementary files for
}

\section{GRACE Water Storage Estimates for the Middle East and Other Regions with Significant Reservoir and Lake Storage}

\section{Submitted to HESS \\ In discussion in HESSD}

Longuevergne L. ${ }^{1}$; Wilson, C.R. ${ }^{2}$; Scanlon, B.R. ${ }^{3}$; Cretaux, J.F. ${ }^{4}$

(1) CNRS - Géosciences Rennes UMR 6118, Université de Rennes 1, Rennes, France

(2) Department of Geological Sciences, Jackson School of Geosciences, The University of Texas at Austin, Austin, TX, USA

(3) Bureau of Economic Geology, Jackson School of Geosciences, The University of Texas at Austin, Austin, TX, USA

(4) CNES/LEGOS, 14, av. Édouard-Belin, 31400 Toulouse, France

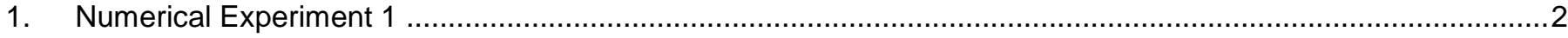

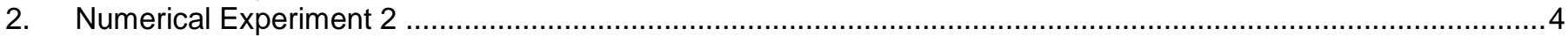

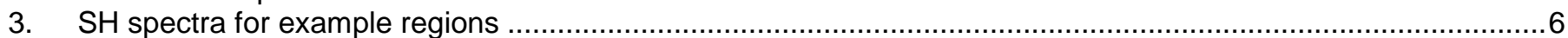

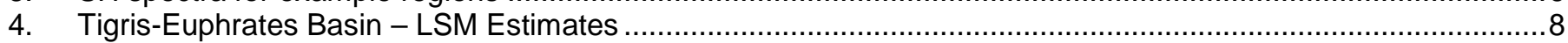

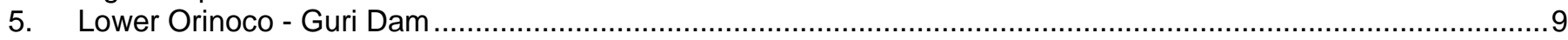

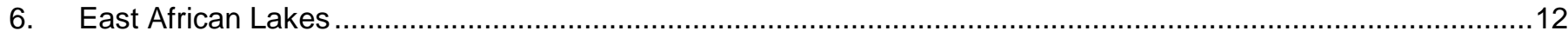




\section{Numerical Experiment 1}

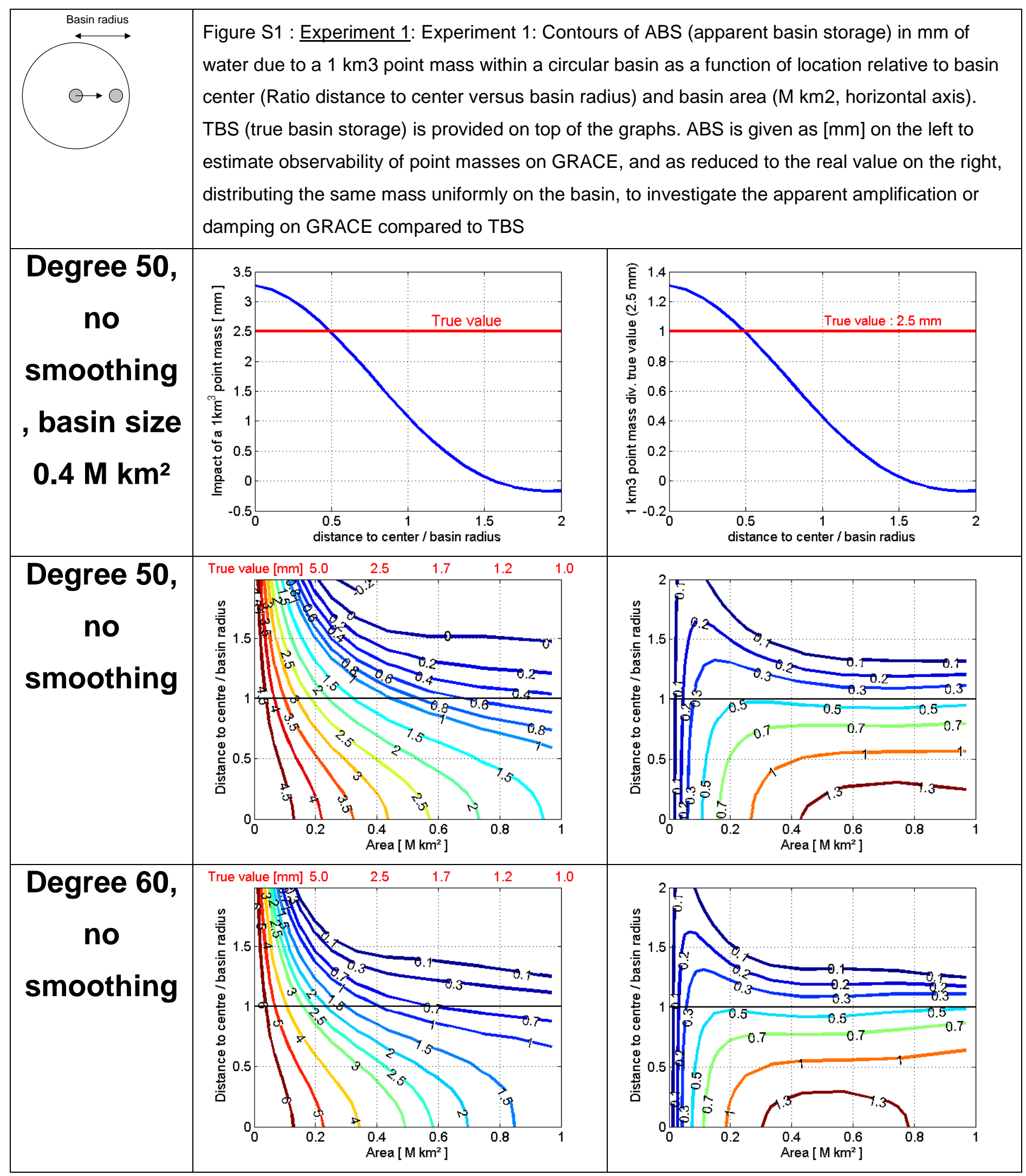




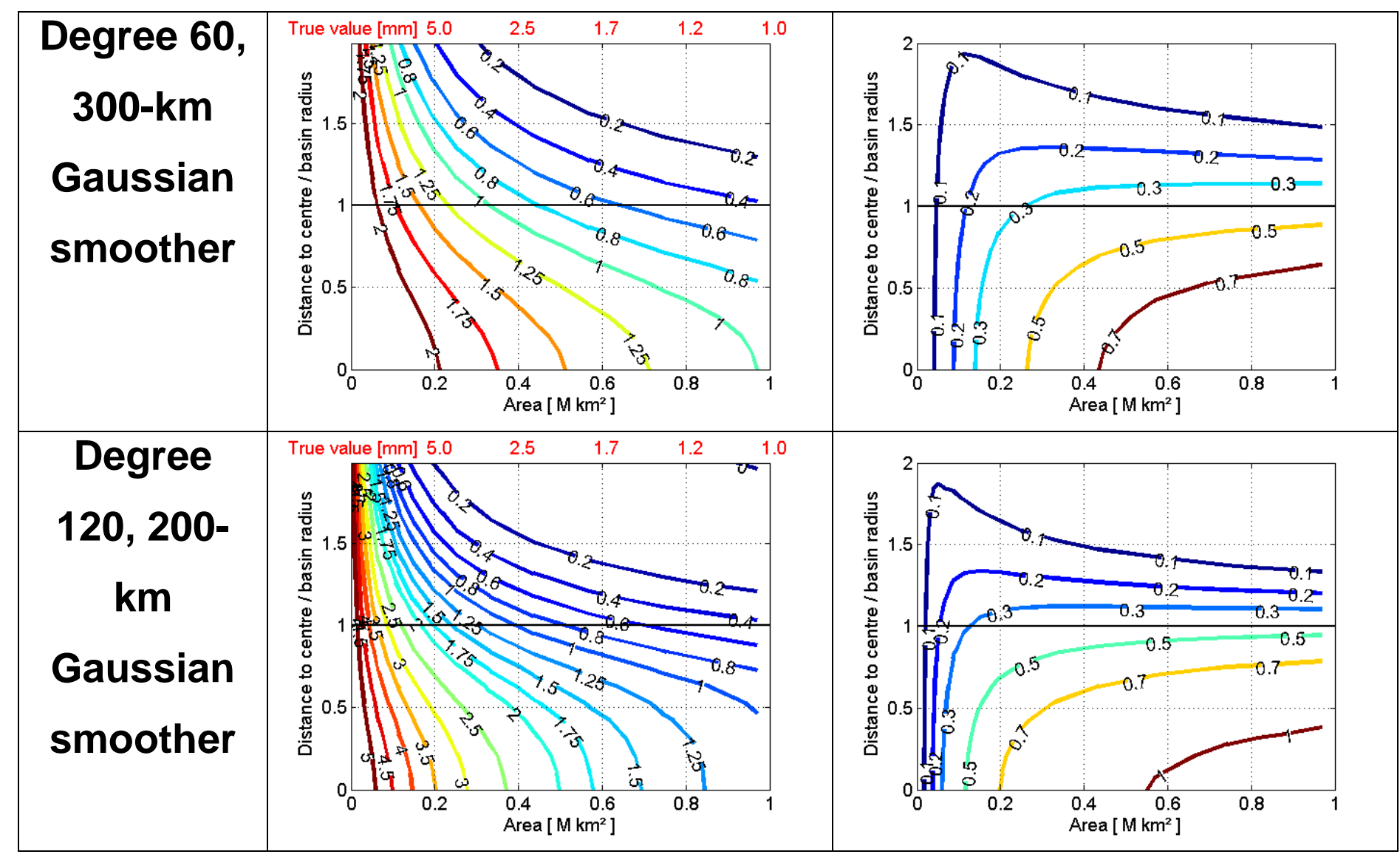




\section{Numerical Experiment 2}

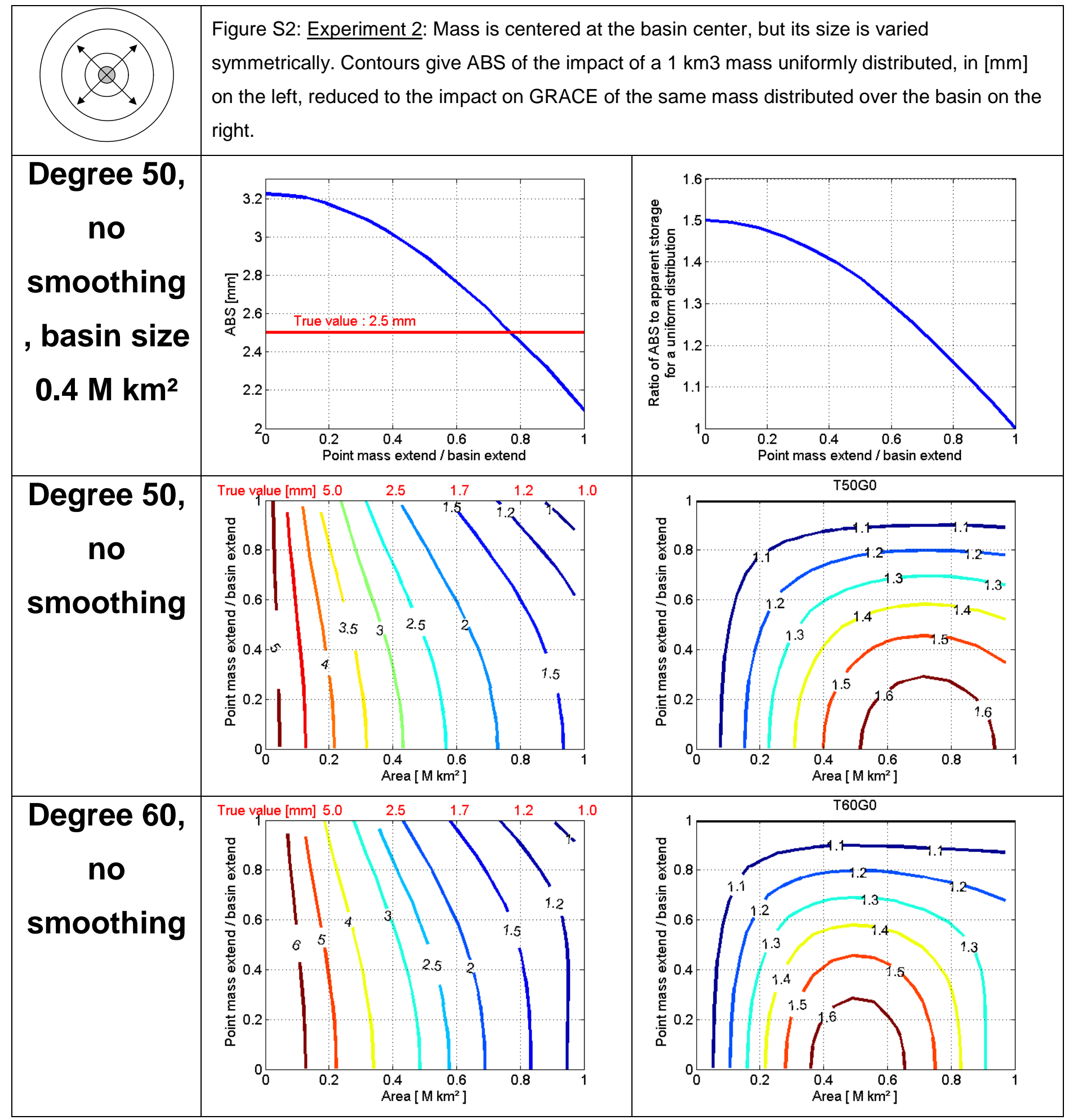




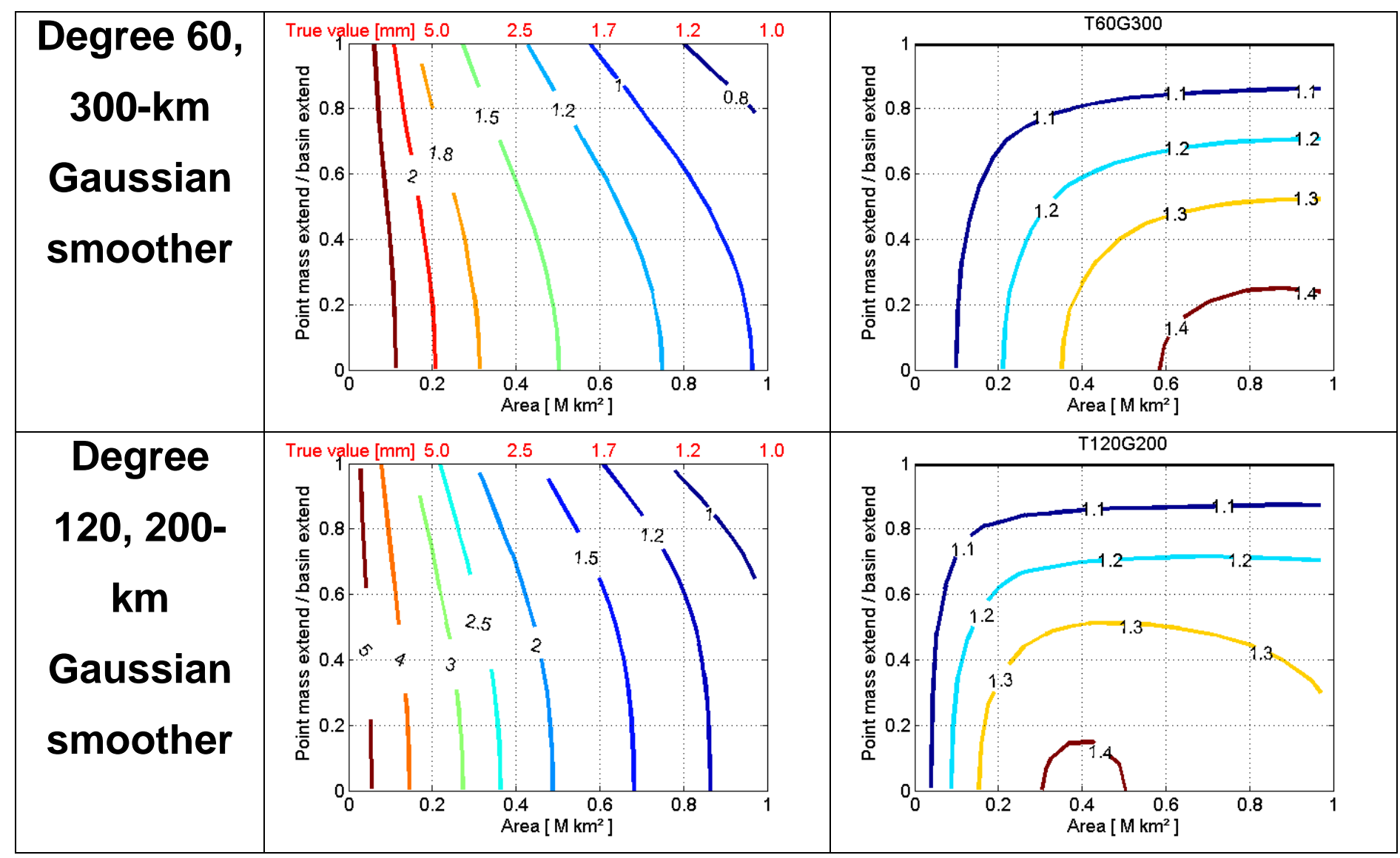




\section{SH spectra for example regions}

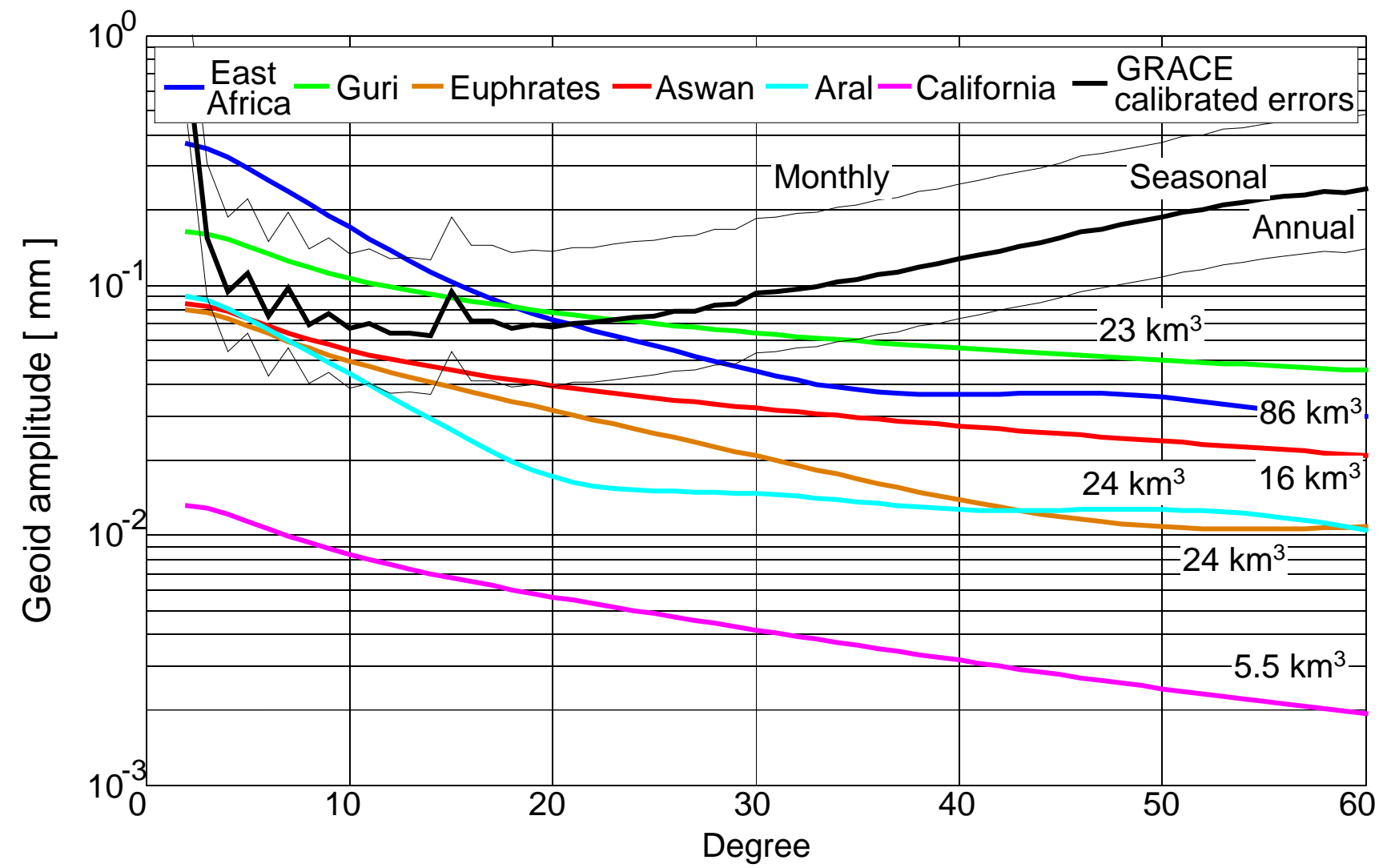

Figure S3: Spherical harmonic $(\mathrm{SH})$ degree-amplitude spectra for GRACE calibrated errors and reservoir storage for the example basins. "Seasonal" and "Annual" calibrated errors are estimated from the decimation process. Lake volume variability is also indicated for each basin. For the California case refer to (Scanlon et al., 2012a). The signature of concentrated masses is evident in the $\mathrm{SH}$ domain where a point mass (impulse) contains all harmonics with equivalent amplitude. Because of the small spatial extend of the reservoir, the amplitude spectrum is decreasing slowly with the harmonic degree 
Table S1: Lake and Reservoir Data Summary.

\begin{tabular}{|c|c|c|c|c|}
\hline Region & Lake name & Area $\left[\mathrm{km}^{2}\right]$ & $\begin{array}{c}\text { Variability } \\
{\left[\mathrm{km}^{3}\right]}\end{array}$ & $\begin{array}{l}\text { Volume / } \\
\text { Level data }\end{array}$ \\
\hline Lower Nile $\left(410000\right.$ km²) $^{2}$ & Aswan & $6216 \mathrm{~km}^{2}$ & $16 \mathrm{~km}^{3}$ & Level \\
\hline Lower Orinoco $\left(350000 \mathrm{~km}^{2}\right)$ & Guri & $3919 \mathrm{~km}^{2}$ & $23 \mathrm{~km}^{3}$ & \\
\hline \multirow[t]{10}{*}{ Tigris-Euphrates (790 000 km²) } & TOTAL & 15268 km² $^{2}$ & $24 \mathrm{~km}^{3}$ & \\
\hline & Asad & 447 & 0.30 & Level \\
\hline & Ataturk & 707 & 1.6 & Level \\
\hline & Daryace & 5200 & 3.1 & Level \\
\hline & Mossoul & 285 & 1.7 & Level \\
\hline & Qadisiyah & 415 & 2.8 & Volume \\
\hline & Razazah & 1501 & 1.3 & Level \\
\hline & Saksak & 458 & 1.6 & Volume \\
\hline & Tharthar & 2500 & 8.5 & Level \\
\hline & Van & 3755 & 1.0 & Level \\
\hline \multirow[t]{14}{*}{ East Africa (1 $125000 \mathrm{~km}^{2}$ ) } & TOTAL & 175144 km $^{2}$ & $86 \mathrm{~km}^{3}$ & \\
\hline & Albert & 5270 & 2.9 & Level \\
\hline & Bangwelu & 9840 & 3.9 & Level \\
\hline & Cahora Bassa & 2739 & 5.6 & Level \\
\hline & Edouard & 2150 & 0.48 & Volume \\
\hline & Kariba & 5400 & 10.6 & Level \\
\hline & Kivu & 2700 & 0.63 & Level \\
\hline & Kyoga & 1720 & 1.2 & Level \\
\hline & Malawi & 29500 & 14 & Level \\
\hline & Mweru & 5120 & 3.0 & Level \\
\hline & Rukwa & 2600 & 2.3 & Level \\
\hline & Tanganyka & 32900 & 12 & Level \\
\hline & Turkana & 6405 & 4.6 & Level \\
\hline & Victoria & 68800 & 25 & \\
\hline \multirow[t]{10}{*}{ Amu - Syr Darya (1 180000 km²) } & TOTAL & 39316 km² & 24 km3 & \\
\hline & Aral sea - North & 3300 & 2.5 & Volume \\
\hline & Aral sea - South & 3000 & 2.6 & Volume \\
\hline & Aydarkul & 3000 & 2.4 & Level \\
\hline & Balkhash & 16996 & 3.9 & Level \\
\hline & Chardarya & 250 & 0.55 & Volume \\
\hline & Issykkul & 6286 & 0.80 & Level \\
\hline & Kapchagayskope & 1200 & 0.56 & Level \\
\hline & Sarykamish & 5000 & 5.3 & Level \\
\hline & Toktogul & 284 & 5.5 & Volume \\
\hline
\end{tabular}


4. Tigris-Euphrates Basin - LSM Estimates

Table S2: Comparison between GRACE, $\triangle \mathrm{SMS}$ and $\triangle R E S$ for Tigris-Euphrates basin. In all cases, adding predicted $\triangle R E S$ contribution to LSM output drive to a better agreement with GRACE data

\begin{tabular}{|c|c|c|c|c|c|c|c|c|c|c|c|}
\hline & $\begin{array}{c}\text { GRACE } \\
\text { CSR }\end{array}$ & CLM & $\begin{array}{c}\text { CLM } \\
+ \\
\text { ARES }\end{array}$ & MOSAIC & $\begin{array}{l}\text { MOSAIC } \\
+\triangle \mathrm{RES}\end{array}$ & $\mathrm{NOAH}$ & $\begin{array}{c}\mathrm{NOAH} \\
+ \\
\triangle \mathrm{RES}\end{array}$ & VIC & $\begin{array}{l}\text { VIC + } \\
\Delta \text { RES }\end{array}$ & WGHM & $\begin{array}{c}\text { WGHM } \\
+ \\
\text { DRES }\end{array}$ \\
\hline $\begin{array}{c}\text { Amplitude of } \\
\text { seasonal } \\
\text { variations } \\
{[\mathrm{mm}]}\end{array}$ & 63.1 & 22.7 & 23.4 & 56.6 & 62.9 & 44.2 & 45.2 & 47.7 & 49.1 & 39.7 & 40.8 \\
\hline $\begin{array}{c}\text { Phase } \\
\text { (seasonal) } \\
\text { [ days ] }\end{array}$ & Ref. & -36 & -15 & -16 & -1.2 & -29 & -17 & -26 & -16 & -29 & -16 \\
\hline Correlation & Ref. & 0.80 & 0.89 & 0.93 & 0.95 & 0.88 & 0.93 & 0.82 & 0.93 & 0.86 & 0.92 \\
\hline $\begin{array}{c}\text { RMS with } \\
\text { GRACE } \\
{[\mathrm{mm}]}\end{array}$ & Ref. & 47 & 38 & 25 & 20 & 34 & 25 & 37 & 27 & 36 & 28 \\
\hline $\begin{array}{c}\text { Trend } \\
(2002 / 10- \\
2009 / 09) \\
\text { [ mm/year ] }\end{array}$ & -11 & -3.2 & -7.3 & -8.4 & -12 & -6.0 & -11 & -3.2 & -7.3 & -5.2 & -9.2 \\
\hline $\begin{array}{c}\text { Trend } \\
(2006 / 10- \\
2009 / 09) \\
\text { [ mm/year ] }\end{array}$ & -39 & -7 & -26 & -21 & -39 & -18 & -37 & -7.9 & -27 & -14 & -33 \\
\hline
\end{tabular}




\section{Lower Orinoco - Guri Dam}

a)

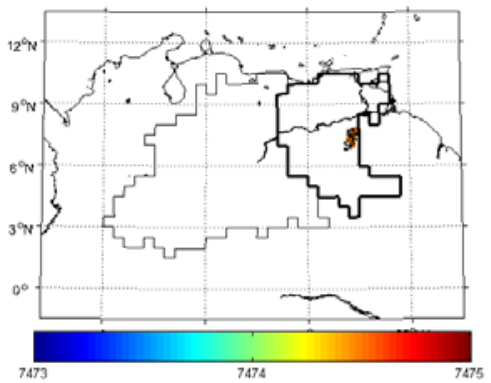

b)

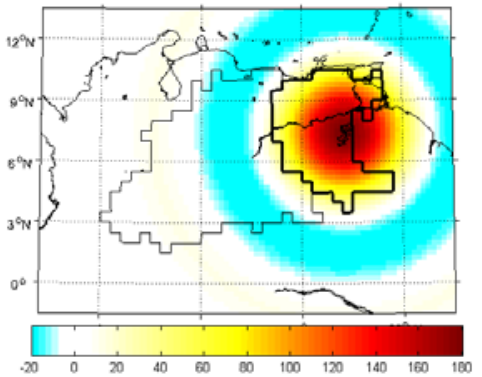

c)

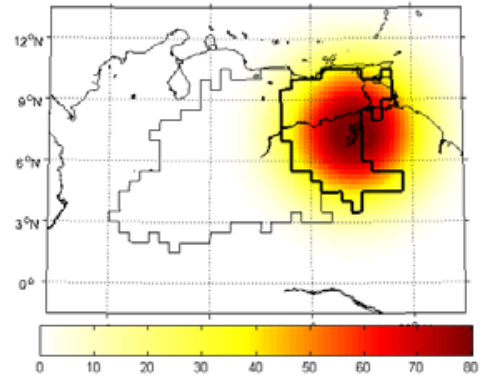

d) Orinoco basin $\left(1 \mathrm{M} \mathrm{km}^{2}\right)$

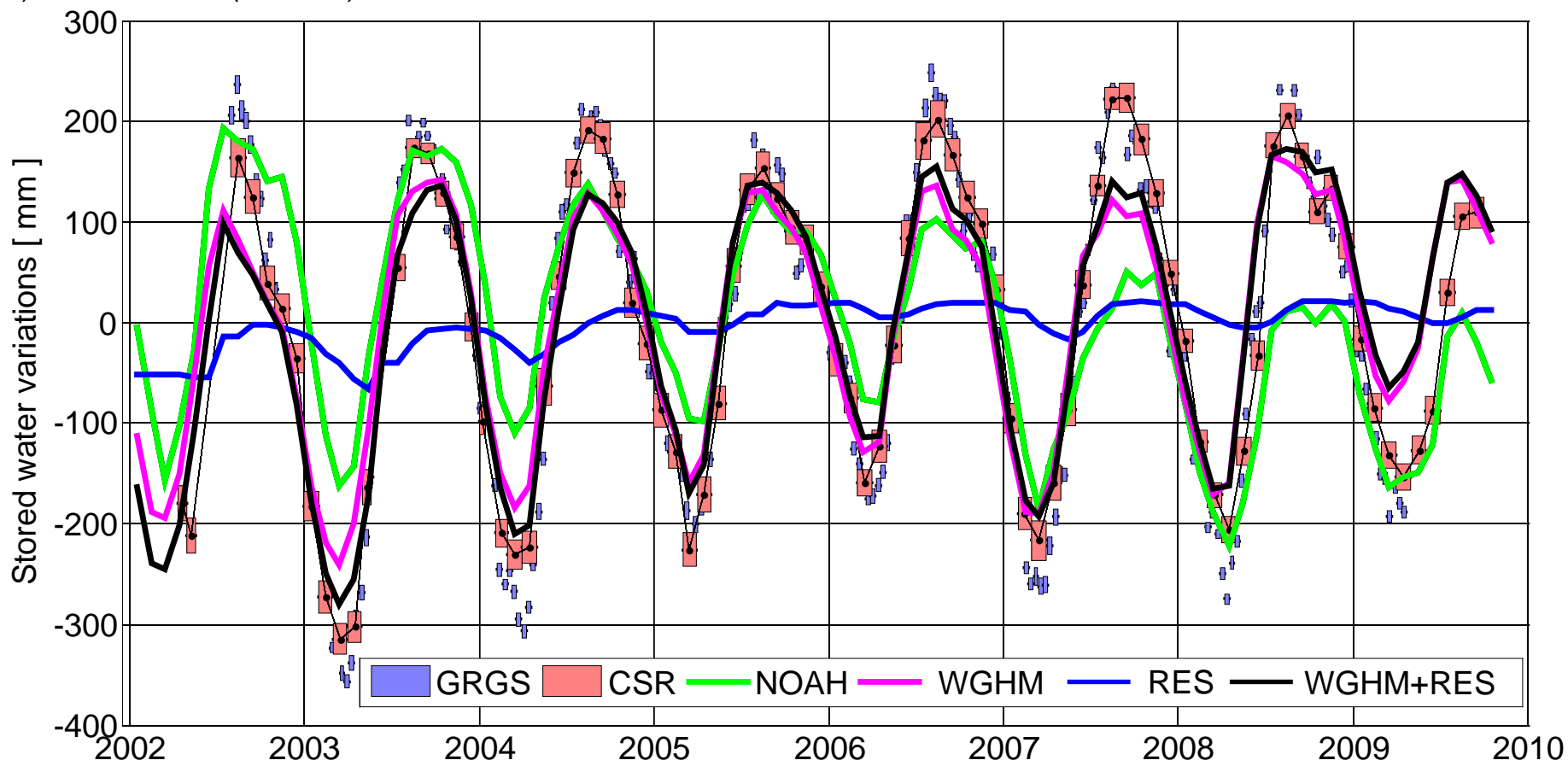

e) Lower Orinoco basin $\left(350000 \mathrm{~km}^{2}\right)$ 


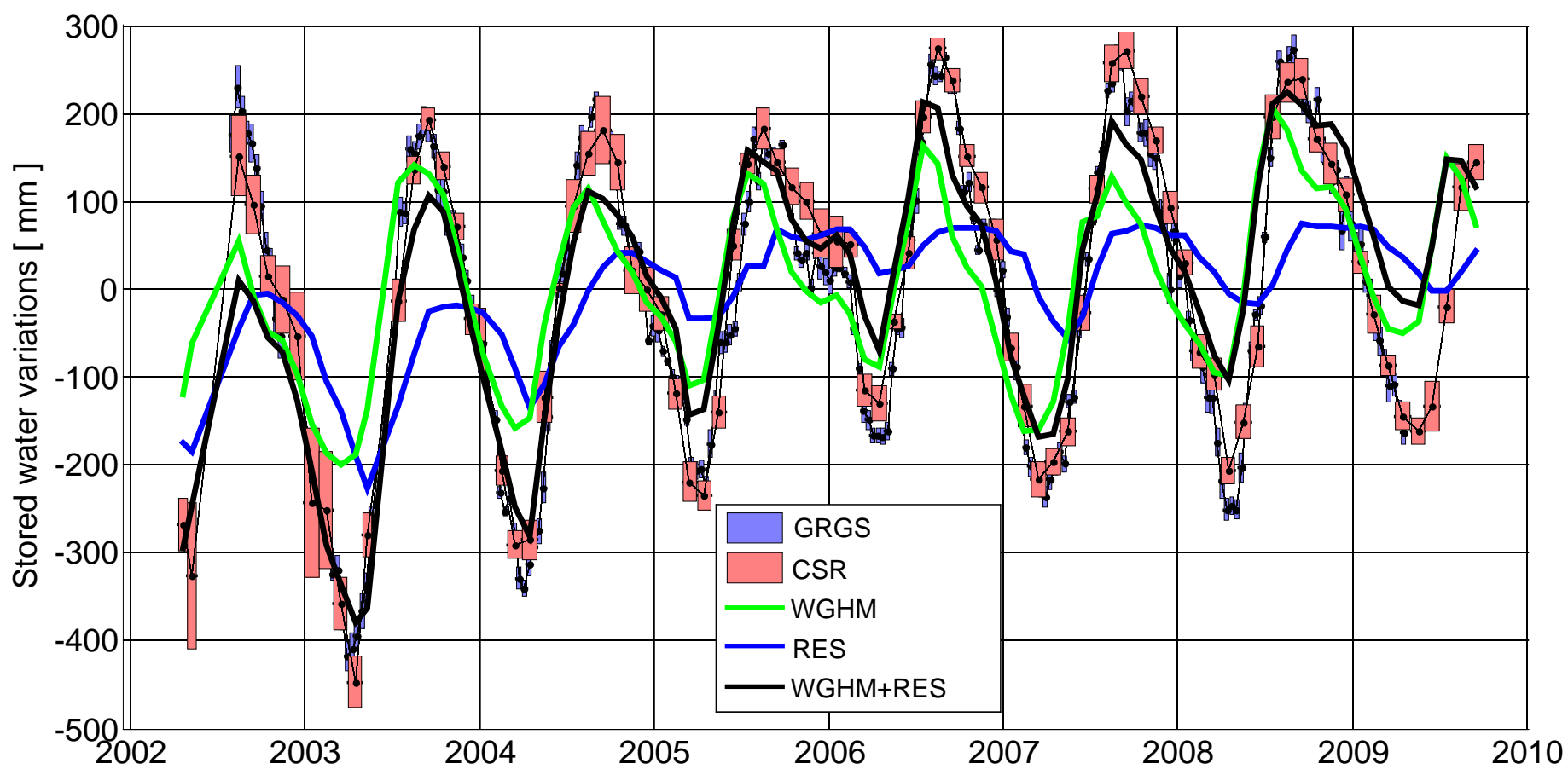

f) Lower Orinoco Basin $\left(350000 \mathrm{~km}^{2}\right)$

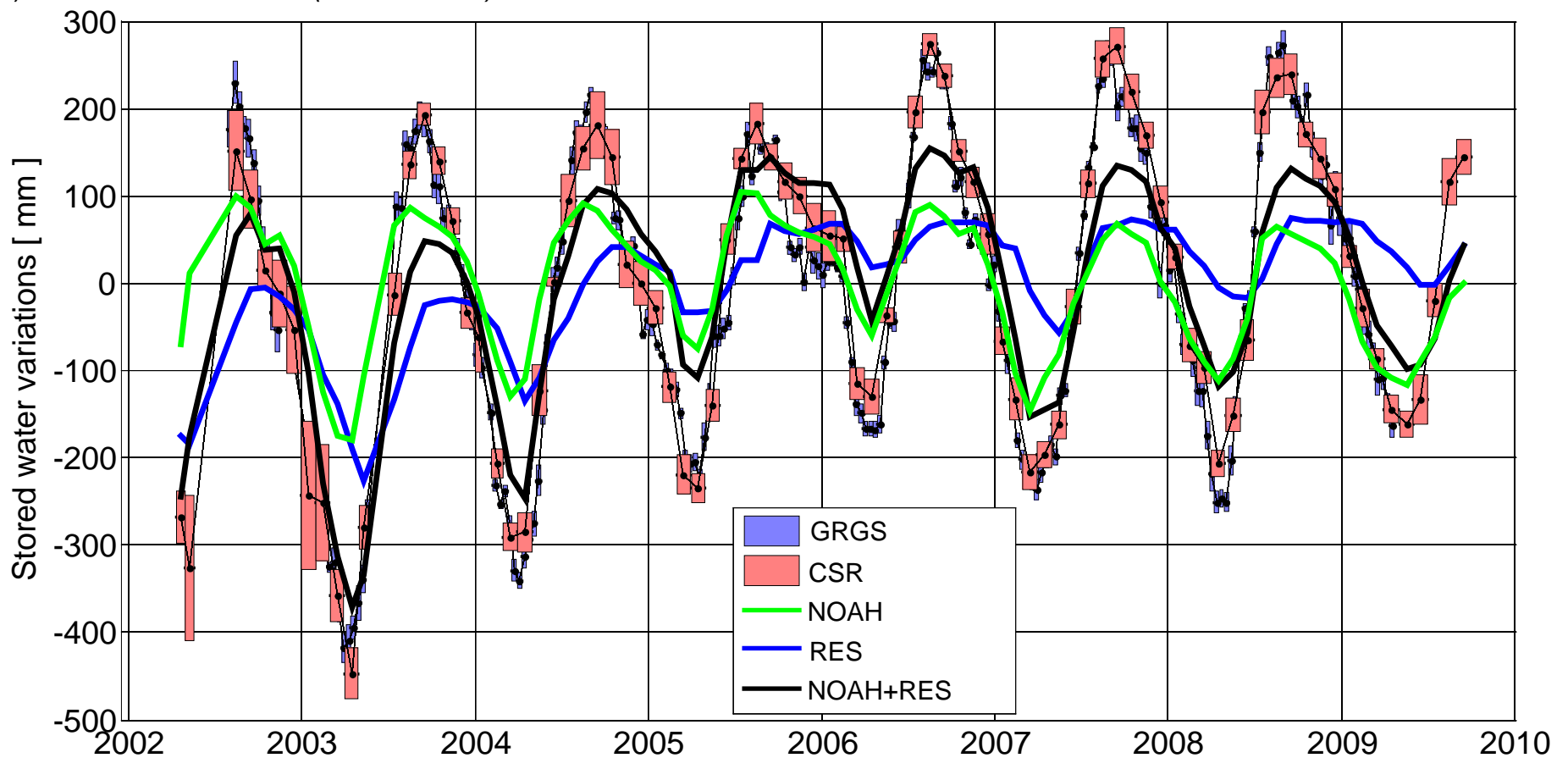

Figure S4: Lower Orinoco and Guri lake contribution. The Guri dam impounds the Caroni River (Venezuela) and creates a lake of $3919 \mathrm{~km}^{2}$. The first stage of the facility was completed in 1969 and completed in 1986, as a 162-m high earth and rockfill dam with a crest length of $11 \mathrm{~km}$. It is mainly used for hydropower purpose (capacity of 10300 $\mathrm{MW}$ ) and covers $50 \%$ of Venezuela's electricity. (a) Reservoir distribution and standard deviation of Guri lake level variation within the basin, (b) same map after truncation at degree 50, (c) same map after truncation at degree 60 and $300-\mathrm{km}$ Gaussian smoother applied. Thick line is lower Orinoco basin (d) GRACE Water storage variations in the Orinoco basin (1 $\mathrm{M} \mathrm{km}^{2}$ ) compared to GLDAS, WGHM and RES from Guri dam, (e) GRACE water storage variations in the Lower Orinoco basin (320 $000 \mathrm{~km}^{2}$ ) compared to WGHM (f) same as e but comparison with NOAH model. Water mass variations from the Inundated area along the course of the river are not modeled in GLDAS and may explain remaining discrepancy with GRACE. 
Table S2: Comparison between GRACE, $\triangle$ SMS and $\triangle R E S$ for Lower Orinoco basin (350 $\left.000 \mathrm{~km}^{2}\right)$

\begin{tabular}{|c|c|c|c|c|c|c|}
\hline & $\begin{array}{c}\text { GRACE } \\
\text { CSR }\end{array}$ & $\begin{array}{c}\Delta \text { RES } \\
\text { Contribution }\end{array}$ & NOAH & $\begin{array}{c}\text { NOAH + } \\
\Delta \text { RES }\end{array}$ & WGHM & $\begin{array}{c}\text { WGHM + } \\
\Delta \text { RES }\end{array}$ \\
\hline Seasonal amplitude [mm] & 199 & $51(25 \%)$ & 85 & $\mathbf{1 2 9}$ & 116 & $\mathbf{1 4 3}$ \\
\hline Phase (seasonal) [ days ] & Ref. & +39 & -14 & $\mathbf{0}$ & -28 & $\mathbf{- 8}$ \\
\hline Correlation & Ref. & & 0.87 & $\mathbf{0 . 9 3}$ & 0.84 & $\mathbf{0 . 9 1}$ \\
\hline Trend [ mm/year ] & 22 & 20 & -4.6 & $\mathbf{1 6}$ & 16 & $\mathbf{3 6}$ \\
\hline
\end{tabular}

85 Table S3: Comparison between GRACE, $\triangle \mathrm{SMS}$ and $\triangle R E S$ for Orinoco basin $\left(1 \mathrm{M} \mathrm{km}^{2}\right)$

\begin{tabular}{|c|c|c|c|c|c|c|}
\hline & $\begin{array}{c}\text { GRACE } \\
\text { CSR }\end{array}$ & $\begin{array}{c}\Delta \text { RES } \\
\text { Contribution }\end{array}$ & NOAH & $\begin{array}{c}\text { NOAH + } \\
\Delta \text { RES }\end{array}$ & WGHM & $\begin{array}{c}\text { WGHM + } \\
\Delta \text { RES }\end{array}$ \\
\hline Seasonal amplitude [mm] & 185 & $16.5(9 \%)$ & 113 & $\mathbf{1 2 7}$ & 146 & $\mathbf{1 5 6}$ \\
\hline Phase (seasonal) [ days ] & Ref. & +44 & 7 & $\mathbf{1 1}$ & -10 & $\mathbf{- 5}$ \\
\hline Correlation & Ref. & & 0.78 & $\mathbf{0 . 8 3}$ & 0.93 & $\mathbf{0 . 9 5}$ \\
\hline Trend [ mm/year ] & 8.1 & 5.3 & -23 & $\mathbf{- 1 8}$ & 10 & $\mathbf{1 6}$ \\
\hline
\end{tabular}


a)

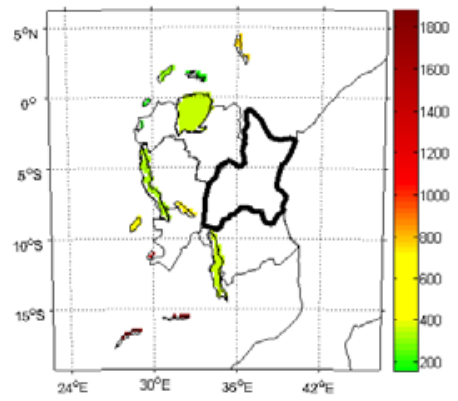

b)

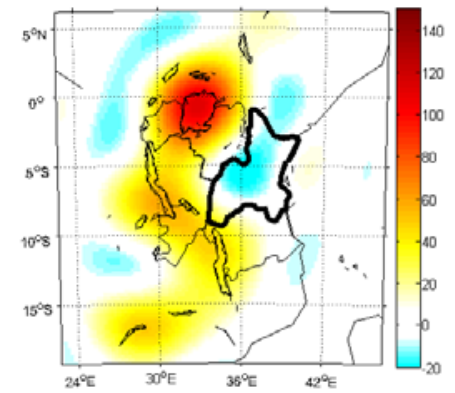

c)

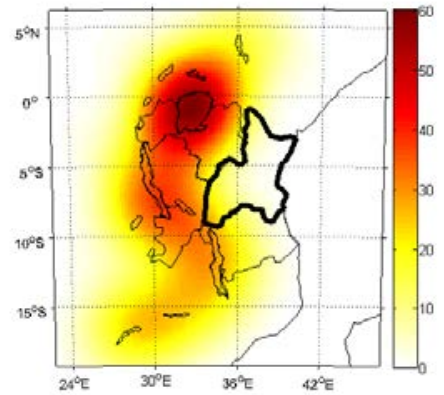

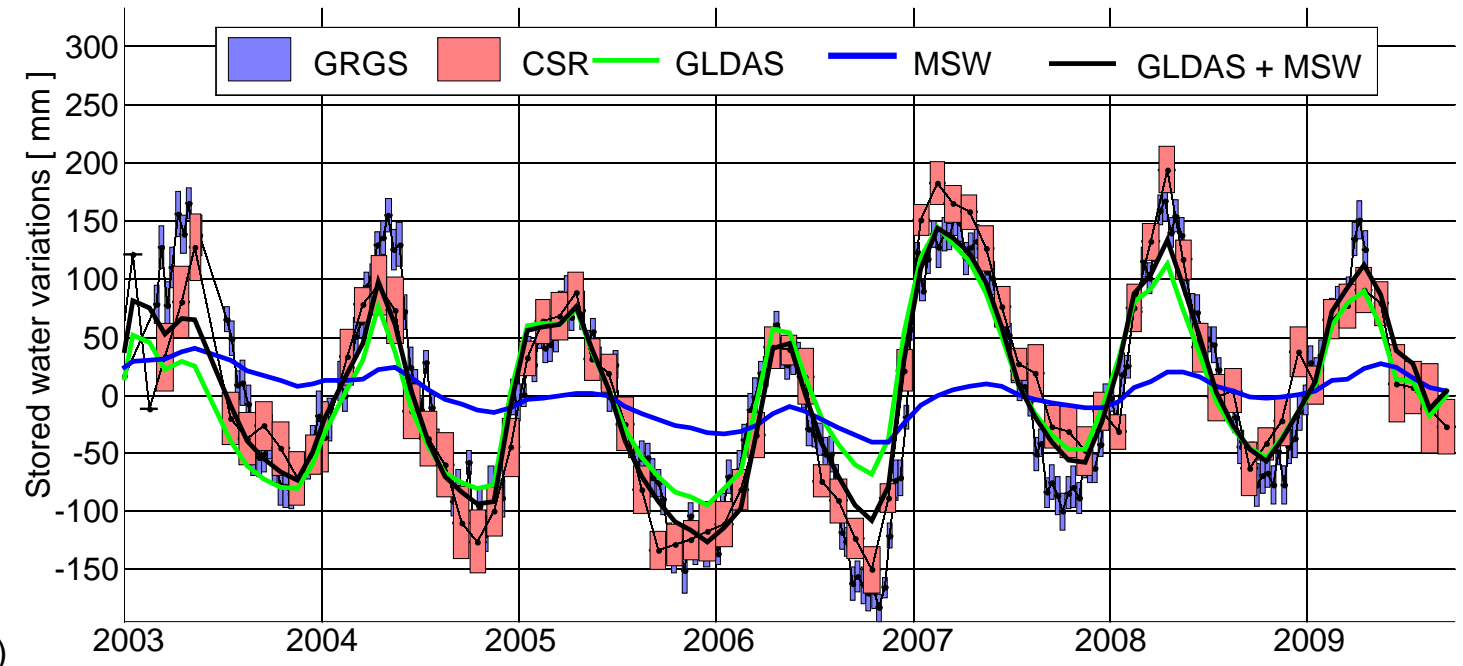

d)

Figure S5: This example explains lake contributions from GRACE from Xie et al.,[ 2012]. (a) reservoir distribution and mass variations for each of the 34 lakes, (b) associated mass variations after truncation to degree 50, (c) mass variations after truncation at degree 60 with $300-\mathrm{km}$ Gaussian smoothing. Thick outline is the North Tanzania coastal basin $\left(355000 \mathrm{~km}^{2}\right.$ ). Note that the predicted lake effect on CSR and GRGS shows different leakage amplitudes and signs in this basin. (d) comparison between lake storage, GLDAS NOAH and GRACE on Tanzania (1 $\left.125000 \mathrm{~km}^{2}\right)$. 